\title{
Pairing with polarization effects in low-density neutron matter
}

\author{
H.-J. Schulze, A. Polls, and A. Ramos \\ Departament d'Estructura i Constituents de la Matèria, Universitat de Barcelona, Av. Diagonal 647, E-08028 Barcelona, Spain
}

(Received 27 October 2000; published 12 March 2001)

\begin{abstract}
We study the properties of the ${ }^{1} S_{0}$ pairing gap in low-density neutron matter. Different corrections to the lowest-order scattering length approximation are explored, resulting in a strong suppression with respect to the BCS result.
\end{abstract}

DOI: 10.1103/PhysRevC.63.044310

\section{INTRODUCTION}

The problem of the influence of the medium on the effective pairing interaction in nuclear matter is a long standing one that still awaits satisfactory solution. This is true even for the simplest case of pairing in the ${ }^{1} S_{0}$ channel in pure neutron matter, to which this article is restricted. A quantitative control on this issue would be very useful in particular for the understanding of neutron star physics [1], where several physical phenomena (cooling, glitches) are thought to depend very sensitively on the size and the density dependence of the gap.

In several publications [2-4] the gap equation is solved in the simplest (BCS) approximation [5-10], namely using the bare neutron-neutron potential as interaction kernel. With a realistic nucleon-nucleon potential, adapted to the scattering phase shifts, one obtains typically a maximum of the gap $\Delta\left(k_{F}\right) \approx 3 \mathrm{MeV}$ at a density corresponding to $k_{F} \approx 0.85$ $\mathrm{fm}^{-1}$.

However, the use of the bare potential completely disregards the influence of the surrounding neutron medium and some authors have attempted to go beyond this level by considering certain additional subsets of diagrams in the interaction kernel [3,11-15]. Unfortunately, doing so the interaction becomes rather complex, and therefore always certain approximations (phase space averages, weak-coupling approximation, ... ) have to be performed in order to arrive at a numerically feasible level. It is well known, however, that the solution of the gap equation depends exponentially on the strength of the interaction, so that any kind of approximation has to be introduced with great care. Also the choice of a particular subseries of graphs has to be considered in this light. Nevertheless, the previous works agree in predicting an important suppression of the pairing gap. However, the precise quantitative level of this suppression as well as its density dependence vary substantially with the different approaches and must be considered unknown for the time being. An overview of the previous results can be found in Ref. [3], for example.

This is the motivation to attempt in this article to face the problem from a different viewpoint, namely a systematic, perturbative treatment valid at low density. The number of further approximations at this level should be kept to a strict minimum. In this article we present the first step within such an approach. More precisely, we will extend the lowest-order interaction kernel by the complete set of diagrams of second order in the interaction and containing one hole line.
PACS number(s): 21.65.+f, 74.20.Fg, 24.10.Cn

In this way we will be able to confirm numerically and to go beyond the well-known asymptotic behavior of the gap, namely within the BCS description $[3,16,17]$

$$
\Delta\left(k_{F}\right) \stackrel{k_{F} \rightarrow 0}{\longrightarrow} \Delta_{0}\left(k_{F}\right)=\frac{8}{e^{2}} \frac{k_{F}^{2}}{2 m} \exp \left[\frac{\pi}{2 k_{F} a_{n n}}\right]
$$

and including polarization effects $[16,18]$

$$
\Delta\left(k_{F}\right) \stackrel{k_{F} \rightarrow 0}{\longrightarrow} \frac{1}{(4 e)^{1 / 3}} \Delta_{0}\left(k_{F}\right),
$$

where $a_{n n}=-18.8 \mathrm{fm}$ is the neutron-neutron scattering length.

Note that this is a surprising result in that, even though the ratio of in-medium and bare interaction becomes unity in the low-density limit, the ratio of the two corresponding gaps does not, but approaches $(4 e)^{-1 / 3} \approx 0.45$. This is due to the nonanalytical dependence of the gap on the interaction, as expressed in Eq. (1a), and will be explained in more detail later on. Stated otherwise, the BCS approximation (using the bare potential) does not yield the correct low-density behavior, but polarization effects are encountered at any density. Of course, Eqs. (1) represent only the asymptotic behavior for $k_{F} \rightarrow 0$, and the purpose of our report is to study numerically the actual gap at finite density, using different approximations for the polarization interaction.

Let us finally mention that these considerations and Eqs. (1) apply only to systems with negative scattering length, such as neutron matter. In the case of a positive scattering length, either there is no gap, or a transition from pairing to bound state formation and Bose-Einstein condensation takes place at sufficiently low density [19], in which case a different expression holds instead of Eq. (1a) [20].

\section{FORMALISM}

We begin with the four-dimensional gap equation [6-8]

$$
\Delta^{*}(K)=i \int \frac{d^{4} K^{\prime}}{(2 \pi)^{4}}\left\langle K^{\prime}|\Gamma| K\right\rangle F^{\dagger}\left(K^{\prime}\right)
$$

with the four-vector $K=\left(k_{0}, \boldsymbol{k}\right)$ and the anomalous propagator 


$$
F^{\dagger}(K)=\frac{-\Delta^{*}(K)}{\left[k_{0}-\epsilon(+K)+i 0\right]\left[k_{0}+\epsilon(-K)-i 0\right]-|\Delta(K)|^{2}},
$$

where $\epsilon(K)=\boldsymbol{k}^{2} / 2 m+\Sigma\left(k_{0}, \boldsymbol{k}\right)-\mu$, and $\mu$ is the neutron chemical potential. $\Gamma$ in Eq. (2) is the complete particleparticle irreducible interaction kernel, itself ultimately involving anomalous propagators. This level of selfconsistency has never been achieved in practice, since it presents a formidable problem.

We will also adhere to this approximation and adopt in this article an even stronger one, namely we disregard the energy dependence of the interaction kernel $\Gamma$ as well as of the neutron self-energy $\Sigma$. This difficult problem [7] will be delayed to future work. Doing so, one arrives at the usual three-dimensional gap equation, involving an "on-shell" interaction kernel $\left\langle\boldsymbol{k}^{\prime}|\boldsymbol{\Gamma}| \boldsymbol{k}\right\rangle$ and neutron single-particle energy $e(k)$. Focusing on the ${ }^{1} S_{0}$ partial wave, the resulting equations are

$$
\begin{array}{r}
\Delta(k)= \\
-\frac{1}{4 \pi^{2}} \int_{0}^{\infty} d k^{\prime} k^{\prime 2} \Gamma{ }_{S_{0}}\left(k, k^{\prime}\right) \frac{\Delta\left(k^{\prime}\right)}{\sqrt{\left[e\left(k^{\prime}\right)-\mu\right]^{2}+\Delta\left(k^{\prime}\right)^{2}}}, \\
\rho=\frac{k_{F}^{3}}{3 \pi^{2}}=\frac{1}{\pi^{2}} \int_{0}^{\infty} d k k^{2} \frac{1}{2}\left[1-\frac{[e(k)-\mu]}{\sqrt{[e(k)-\mu]^{2}+\Delta(k)^{2}}}\right],
\end{array}
$$

determining the gap function $\Delta(k)$ and the chemical potential $\mu$ for a given neutron density $\rho$ or neutron Fermi momentum $k_{F}$. It should be remembered that the consistent choice for the neutron single-particle energy $e(k)$ within the mean-field BCS approach $(\Gamma=V)$ is the Hartree-Fock approximation $[7,10]$. However, at very low density the effect is very small and a kinetic spectrum $e(k)=k^{2} / 2 m$ can be used instead. Also the two equations above can be decoupled by setting $\mu=e\left(k_{F}\right)$ in this case (if the scattering length is negative).

Regarding the bare interaction $V$, we will use in this work the Argonne $V_{18}$ [21] neutron-neutron potential, which is very well fitted to the $T=1$ scattering phase shifts. For $s$-wave pairing it is sufficient to consider the central components of the potential acting in spin $S=0,1$ channels:

$$
\widetilde{V}(r)=\widetilde{V}_{0}(r) \frac{1-\boldsymbol{\sigma}_{1} \cdot \boldsymbol{\sigma}_{2}}{4}+\widetilde{V}_{1}(r) \frac{3+\boldsymbol{\sigma}_{1} \cdot \boldsymbol{\sigma}_{2}}{4} .
$$

The Fourier transforms in momentum space are then $(S$ $=0,1)$

$$
V_{S}(q)=4 \pi \int_{0}^{\infty} d r r^{2} j_{0}(q r) \tilde{V}_{S}(r),
$$
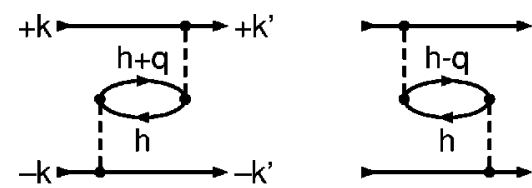

(a)
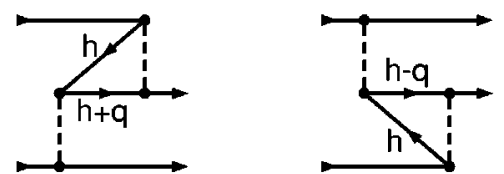

(b)
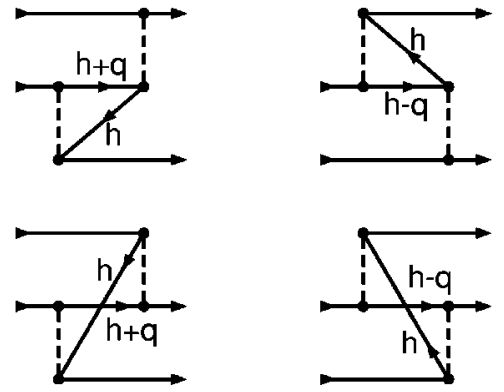

(c)

FIG. 1. Diagrams of second order in the interaction (dashed lines) and with one hole line $h$ contributing to the neutron-neutron interaction kernel.

and the interaction $\Gamma_{1} s_{0}$ in Eq. (4a) to lowest (first) order in the potential is equal to the isotropic part of a partial wave expansion:

$$
\begin{aligned}
& V_{{ } S_{0}}\left(k, k^{\prime}\right)=\frac{1}{2} \int_{-1}^{+1} d z\left\langle\boldsymbol{k}^{\prime}|V| \boldsymbol{k}\right\rangle_{S=0}, \quad z=\hat{\boldsymbol{k}}^{\prime} \cdot \hat{\boldsymbol{k}} \\
& =\frac{1}{2 k k^{\prime}} \int_{\left|k-k^{\prime}\right|}^{k+k^{\prime}} d q q V_{0}(q), q^{2}=k^{2}+k^{\prime 2}-2 k k^{\prime} z .
\end{aligned}
$$

Going now beyond the BCS approximation, we display in Fig. 1 the complete set of diagrams in the particle-particle channel of second order in the interaction and containing one hole line $[16,18,22]$. The interaction appearing internally in these diagrams is in the simplest case the free $T$ matrix, for which we will in the following assume a composition into spin $S=0,1$ components $T_{0}$ and $T_{1}$ in analogy to Eq. (5). The diagrams of type (a) shown in the figure are the usual (ring) polarization graphs, whereas (b) and (c) are the results of performing exchange on one or both potential lines appearing in (a), respectively. It is therefore clear that the complete set is intrinsically connected and must be considered together. The neutron propagators appearing in those diagrams are bare propagators in order to strictly follow the concept of a perturbative low-density expansion and avoiding any selfconsistency.

Denoting the diagrams by $W_{a}, W_{b}$, and $W_{c}$, and taking care of the spin dependence of the interaction, one obtains explicitly: 


$$
\begin{gathered}
\left\langle\boldsymbol{k}^{\prime}\left|W_{a}\right| \boldsymbol{k}\right\rangle_{S=0}=+2 \sum_{h} \frac{f_{h} \bar{f}_{h+q}}{e_{h}-e_{h+q}}\left\langle\boldsymbol{h}+\boldsymbol{q},-\boldsymbol{k}^{\prime}\left|\left(T|\boldsymbol{h},-\boldsymbol{k}\rangle\left\langle\boldsymbol{h}, \boldsymbol{k}^{\prime}\right| T\right)_{a}\right| \boldsymbol{h}+\boldsymbol{q}, \boldsymbol{k}\right\rangle, \\
\left\langle\boldsymbol{k}^{\prime}\left|W_{b}\right| \boldsymbol{k}\right\rangle_{S=0}=-4 \sum_{h} \frac{f_{h} \bar{f}_{h+q}}{e_{h}-e_{h+q}}\left\langle\boldsymbol{h}+\boldsymbol{q},-\boldsymbol{k}^{\prime}\left|\left(T|\boldsymbol{h},-\boldsymbol{k}\rangle\left\langle\boldsymbol{h}, \boldsymbol{k}^{\prime}\right| T\right)_{b}\right| \boldsymbol{k}, \boldsymbol{h}+\boldsymbol{q}\right\rangle, \\
\left\langle\boldsymbol{k}^{\prime}\left|W_{c}\right| \boldsymbol{k}\right\rangle_{S=0}=-2 \sum_{h} \frac{f_{h} \bar{f}_{h+q}}{e_{h}-e_{h+q}}\left\langle\boldsymbol{h}+\boldsymbol{q},-\boldsymbol{k}^{\prime}\left|\left(T|-\boldsymbol{k}, \boldsymbol{h}\rangle\left\langle\boldsymbol{h}, \boldsymbol{k}^{\prime}\right| T\right)_{c}\right| \boldsymbol{k}, \boldsymbol{h}+\boldsymbol{q}\right\rangle,
\end{gathered}
$$

where $\boldsymbol{q}=\boldsymbol{k}^{\prime}-\boldsymbol{k}$, with the Fermi distribution $f_{k}=\theta\left(k_{F}-k\right)$, $\bar{f} \equiv 1-f$, and $e_{k}=k^{2} / 2 m$. The notation

$$
\begin{aligned}
& (T T)_{a}=\frac{1}{4}\left[-T_{0} T_{0}+3 T_{1} T_{1}+3\left(T_{0} T_{1}+T_{1} T_{0}\right)\right], \\
& (T T)_{b}=\frac{1}{4}\left[+T_{0} T_{0}+3 T_{1} T_{1}+3\left(T_{0} T_{1}-T_{1} T_{0}\right)\right], \\
& (T T)_{c}=\frac{1}{4}\left[+T_{0} T_{0}-3 T_{1} T_{1}+3\left(T_{0} T_{1}+T_{1} T_{0}\right)\right]
\end{aligned}
$$

has been introduced for compactness.

The principal practical problem with these expressions is the three-dimensional integration $\Sigma_{h}$ that has to be performed. However, a strong simplification can be achieved by neglecting, in line with the low-density expansion, the hole momentum $\boldsymbol{h}$ in the arguments of the interaction that appear in the equations above. In this case the $h$ integration can be performed analytically, leading to the well-known Lindhard function at zero energy transfer,

$$
\begin{aligned}
4 \sum_{h} \frac{f_{h} \bar{f}_{h+q}}{e_{h}-e_{h+q}}=\Pi(q) & =-\frac{m k_{F}}{\pi^{2}}\left[\frac{1}{2}+\frac{1-x^{2}}{4 x} \ln \left|\frac{1+x}{1-x}\right|\right] \\
x & =q / 2 k_{F} .
\end{aligned}
$$

Now the Lindhard function cuts off also the momentum $\boldsymbol{q}$ appearing in Eqs. (8), so that finally the approximation for the complete second-order interaction kernel becomes

$$
\begin{aligned}
\left\langle\boldsymbol{k}^{\prime}\left|W_{a+b+c}\right| \boldsymbol{k}\right\rangle_{S=0}= & \frac{\Pi(q)}{2}\left[\left\langle\boldsymbol{p}^{\prime}\left|\left(T|\boldsymbol{p}\rangle\left\langle-\boldsymbol{p}^{\prime}\right| T\right)_{a}\right|-\boldsymbol{p}\right\rangle\right. \\
& -2\left\langle\boldsymbol{p}^{\prime}\left|\left(T|\boldsymbol{p}\rangle\left\langle-\boldsymbol{p}^{\prime}\right| T\right)_{b}\right| \boldsymbol{p}\right\rangle \\
& \left.-\left\langle\boldsymbol{p}^{\prime}\left|\left(T|-\boldsymbol{p}\rangle\left\langle-\boldsymbol{p}^{\prime}\right| T\right)_{c}\right| \boldsymbol{p}\right\rangle\right],
\end{aligned}
$$

where for brevity now only the relative momenta $\boldsymbol{p}=\boldsymbol{k} / 2$ and $\boldsymbol{p}^{\prime}=\boldsymbol{k}^{\prime} / 2$ are indicated on the right-hand side (rhs).

In order to obtain the polarization contribution to the interaction in the ${ }^{1} S_{0}$ channel that we denote by $W^{1} S_{0}\left(k, k^{\prime}\right)$, an integration on $z=\hat{\boldsymbol{k}}^{\prime} \cdot \hat{\boldsymbol{k}}$, or equivalently on $q$, as specified in Eq. (7), has to be performed on this expression. For our analysis it is sufficient to consider the leading partial waves in the $S=0,1$ channels of the $T$ matrix, namely the ${ }^{1} S_{0}$ and ${ }^{3} P_{J}$ states, respectively. We have then

$$
\begin{gathered}
\left\langle\boldsymbol{p}^{\prime}\left|T_{0}\right| \boldsymbol{p}\right\rangle=T_{0}\left(p, p^{\prime}\right), \\
\left\langle\boldsymbol{p}^{\prime}\left|T_{1}\right| \boldsymbol{p}\right\rangle=T_{1}\left(p, p^{\prime}\right) 3 z,
\end{gathered}
$$

and obtain the final result for the polarization interaction

$$
\begin{aligned}
W_{{ }^{1} S_{0}}\left(k, k^{\prime}\right)= & -\frac{\Pi_{0}\left(k, k^{\prime}\right)}{2} T_{0}\left(\frac{k}{2}, \frac{k^{\prime}}{2}\right)^{2} \\
& +18 \frac{\Pi_{1}\left(k, k^{\prime}\right)}{2} T_{0}\left(\frac{k}{2}, \frac{k^{\prime}}{2}\right) T_{1}\left(\frac{k}{2}, \frac{k^{\prime}}{2}\right) \\
& +27 \frac{\Pi_{2}\left(k, k^{\prime}\right)}{2} T_{1}\left(\frac{k}{2}, \frac{k^{\prime}}{2}\right)^{2},
\end{aligned}
$$

with the weight functions

$$
\Pi_{i}\left(k, k^{\prime}\right)=\frac{1}{2} \int_{-1}^{+1} d z z^{i} \Pi(q) .
$$

These integrations can be carried out analytically, making use of the integrals

$$
\begin{gathered}
\int_{0}^{y} d x x^{2 j+1}\left[\frac{1}{2}+\frac{1-x^{2}}{4 x} \ln \left|\frac{1+x}{1-x}\right|\right] \\
=\frac{1}{12}\left[2 \ln \left|1-y^{2}\right|+\left(3-y^{2}\right) y \ln \left|\frac{1+y}{1-y}\right|+2 y^{2}\right] \\
(j=0), \\
\frac{1}{60}\left[2 \ln \left|1-y^{2}\right|+\left(5-3 y^{2}\right) y^{3} \ln \left|\frac{1+y}{1-y}\right|+2 y^{2}+6 y^{4}\right] \\
(j=1)
\end{gathered}
$$

$$
\begin{gathered}
\frac{1}{140}\left[2 \ln \left|1-y^{2}\right|+\left(7-5 y^{2}\right) y^{5} \ln \left|\frac{1+y}{1-y}\right|+2 y^{2}+y^{4}+10 y^{6}\right], \\
(j=2) .
\end{gathered}
$$

The weight functions $\Pi_{i}\left(k, k^{\prime}\right), i=0,1$ are displayed in Fig. 2 , from which it can clearly be seen how the Lindhard func- 

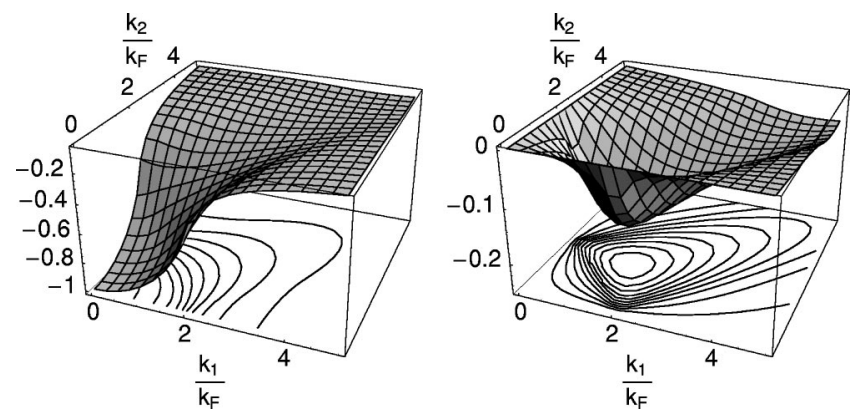

FIG. 2. The weight functions $\left(\pi^{2} / m k_{F}\right) \Pi_{i}\left(k_{1}, k_{2}\right)$, Eq. (14), for $i=0$ (left) and $i=1$ (right).

tion cuts off the interaction in momentum space, so that with vanishing density ultimately only the zero-range value (scattering length) matters. The functions are all negative definite, so that the first and the last term on the rhs of Eq. (13) act always repulsive and attractive, respectively, whereas the effect of the second term depends on the relative sign of $T_{0}$ and $T_{1}$.

We will now use effective range approximations for the phase shifts in the two relevant partial waves,

$$
\begin{gathered}
T_{S}(p)=-\frac{4 \pi}{m p} e^{i \delta_{S} \sin \delta_{S}} \\
\tan \delta_{0}(p) \approx p\left(-\frac{1}{a_{n n}}+\frac{r_{n n}}{2} p^{2}\right)^{-1}, \quad a_{n n}=-18.8 \mathrm{fm} \\
r_{n n}=2.8 \mathrm{fm} \\
\tan \delta_{1}(p) \approx-\left(b_{n n} p\right)^{3}, \quad b_{n n} \approx-1 \mathrm{fm}
\end{gathered}
$$

with the relevant scattering lengths $a_{n n}, b_{n n}$, and effective range $r_{n n}$, respectively. ${ }^{1}$ For our purpose these on-shell parametrizations have to be extrapolated, which is done by setting $p^{2}=\left(k^{2}+k^{\prime 2}\right) / 8$ for the use in Eq. (13).

Since Eq. (16) constitutes an approximation to the $T$ matrix keeping terms up to momenta squared, for consistency the first two terms on the rhs of Eq. (13) above should be considered when constructing the polarization interaction, whereas the third term can be neglected. This is the approximation that we use in the following, although eventually it turns out that even the second term can be neglected as well. We remark at this point that the imaginary part of the $T$ matrix (16a) leads to an imaginary part of the polarization interaction (13), which has to be neglected in the present approximation scheme, since from the beginning the energy dependence of the gap equation was discarded.

\footnotetext{
${ }^{1}$ We use a characteristic value for the $p$-wave scattering length $b_{n n}$, in order to check the influence on the gap that turns out to be very small. The physical scattering lengths are different for the three ${ }^{3} P_{J}$ states: $b_{n n} \approx-1.4,+1.2,-0.7 \mathrm{fm}$ for $J=0,1,2$, respectively.
}

So far we have discussed the construction of the interaction kernel using first-order polarization diagrams embodying the free $T$ matrix. The density dependence of the kernel is therefore solely due to the Lindhard function. However, the scattering matrix $T$ that also determines the polarization interaction, is by itself modified inside the medium. In principle, it is to be replaced by the full particle-hole interaction, to be determined within a fully self-consistent (Babu-Brown) approach [23]. This, however, is at the moment not practically feasible, leading to different approximation schemes found in the literature, as discussed in the Introduction.

In the low-density limit considered here, we can nevertheless try to estimate the consequences of this modification. There are two principal physical effects. The first one is the action of Pauli-blocking in the intermediate states of the $T$ matrix, i.e., the replacement of the $T$ matrix by the Brueckner $G$ matrix. We will attempt to take into account the major effect of this modification by using the $G$-matrix scattering length instead of the bare one in Eq. (16b). The change of the momentum dependence of the $T$ matrix will be neglected, however. The $G$-matrix scattering length $a_{n n}\left(k_{F}\right)$ is displayed in the top panel of Fig. 3, and it can be seen that even in the low-density interval considered, there is some important variation (reduction in size) with increasing density. For details of the $G$-matrix calculation the reader is referred to Ref. [24].

Besides these ladder correlations, the particle-hole interaction is modified by polarization contributions. The leading corrections of this kind carry one hole line (one polarization "bubble"') and have to be considered together with the polarization graphs in the particle-particle channel containing two hole lines. To compute systematically the effect of these polarization graphs in next-to-leading order in density (more than one hole line) is a very hard task [25] and beyond the aim of this paper. We can, however, try to estimate the error that is made by neglecting these contributions.

First, one notes from Eqs. (10) and (16) that the relevant expansion parameter giving the relative magnitude of the polarization diagrams of $(n+1)$ th order, with respect to those of $n$th order is $\sim k_{F} a_{n n}$. Naively, this parameter can be translated into an estimate of the relative accuracy of the gap including only first-order polarization effects in the following manner: We extend in the analytical BCS result (1a) the interaction by terms up to second-order polarization effects:

$$
\Delta\left(k_{F}\right) \stackrel{k_{F} \rightarrow 0}{\longrightarrow} \frac{8}{e^{2}} \frac{k_{F}^{2}}{2 m} \exp \left[\frac{\pi / 2}{\kappa+c_{1} \kappa^{2}\left(1 \pm c_{2} \kappa\right)}\right], \quad \kappa=k_{F} a_{n n}
$$

where

$$
c_{1}=-\frac{2 \pi}{m k_{F}} \Pi_{0}\left(k_{F}, k_{F}\right)=\frac{2}{3 \pi}(1+2 \ln 2) \approx 0.506
$$

accounts for the polarization effects to first order and $c_{2}$ is the unknown parameter (of order unity) corresponding to second-order polarization effects. Expanding now the argu- 


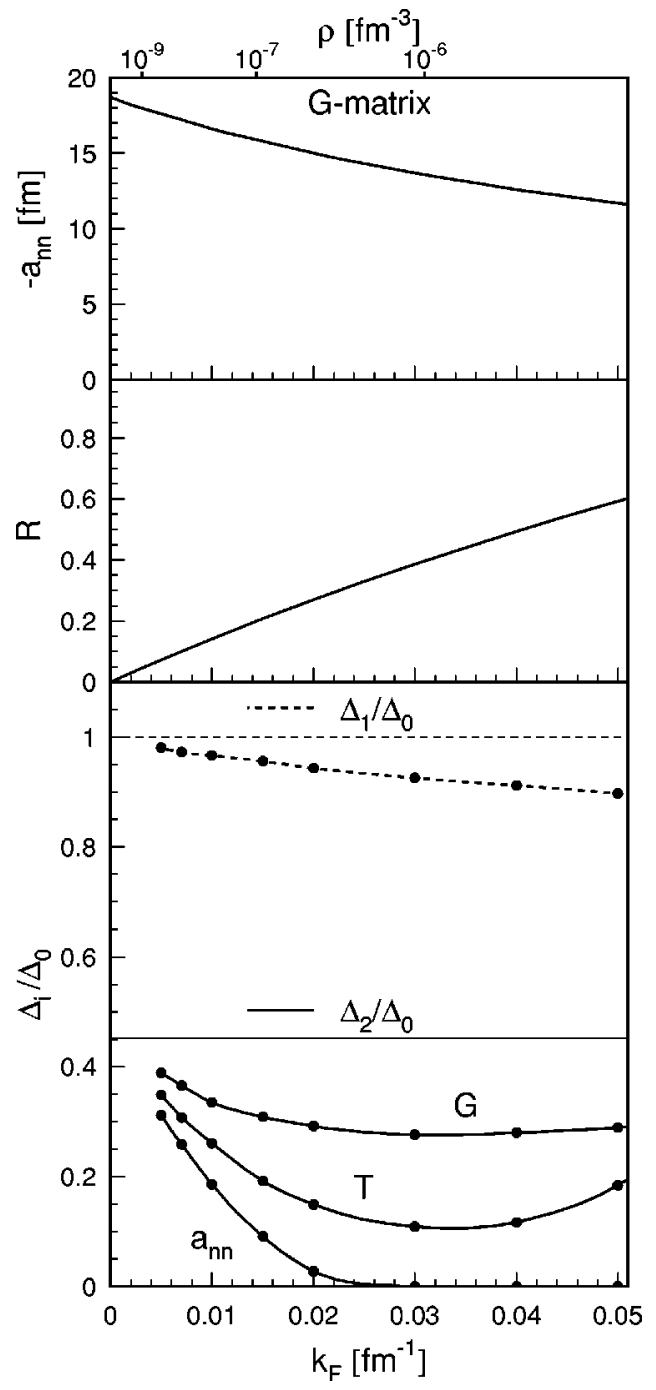

FIG. 3. Top panel: The $G$-matrix scattering length as a function of Fermi momentum. Middle panel: The parameter $R$, Eq. (21), measuring the relative accuracy of the results displayed in the panel below. Bottom panel: ${ }^{1} S_{0}$ gap in neutron matter as a function of Fermi momentum $k_{F}$. Plotted are the ratios $\Delta_{1} / \Delta_{0}$ (dashed curve carrying markers) and $\Delta_{2} / \Delta_{0}$ (solid curves), where $\Delta_{0}$ is given in Eq. (1a), $\Delta_{1}$ is the BCS gap obtained with the first-order bare interaction, and $\Delta_{2}$ is the gap including first-order polarization contributions. In the latter case, different approximations for the interaction are used, as explained in the text. The horizontal dashed and solid lines indicate the values according to Eqs. (1a) and (1b), respectively.

ment of the exponential up to third order in $\kappa$, one obtains for the ratio relative to the $\mathrm{BCS}$ value,

$$
\begin{aligned}
\frac{\Delta\left(k_{F}\right)}{\Delta_{0}\left(k_{F}\right)} & =\exp \left[-\frac{\pi}{2} c_{1}\left[1+\left( \pm c_{2}-c_{1}\right) \kappa+\mathcal{O}\left(\kappa^{2}\right)\right]\right] \\
& \approx\left[\frac{1}{(4 e)^{1 / 3}}\right]^{\left(1-c_{1} \kappa\right)} \times\left[\frac{1}{(4 e)^{1 / 3}}\right]^{ \pm c_{2} \kappa} .
\end{aligned}
$$

Therefore, formula (1b) for the effect of first-order polarization is recovered, while (assuming $\left|c_{2}\right| \lesssim 1$ )

$$
R(\kappa)=(4 e)^{-\kappa / 3}-1
$$

is an estimate of the relative accuracy of this result with respect to inclusion of second-order contributions. We repeat that this "derivation" is only a crude way to roughly estimate the accuracy of our results.

Now that the construction of the interaction kernel has been completed, we can proceed to the numerical evaluation and presentation of the results.

\section{RESULTS}

For the numerical solution of the gap equation (4a), care must be taken in the choice of a suitable grid in momentum space, because the integrand becomes more and more peaked at $k^{\prime}=k_{F}$ when approaching smaller densities. A good test is the comparison of the numerical result in BCS [using the interaction kernel (7)] with the analytical limit (1a). This is shown in the lower panel of Fig. 3 (dashed curve denoted $\left.\Delta_{1} / \Delta_{0}\right)$, and indeed the limit is properly approached.

We can therefore proceed to the inclusion of polarization effects according to Eq. (13). The results for the pairing gap with different approximations for the polarization interaction are displayed in the same plot. (Solid lines denoted $\Delta_{2} / \Delta_{0}$.) The simplest approximation (curve denoted $a_{n n}$ ) corresponds to completely neglecting the momentum dependence of the $T$ matrix, replacing it by a constant, $T_{0}(0,0)=4 \pi a_{n n} / m$. The momentum dependence of the polarization interaction is then solely due to the Lindhard function, Eq. (14) with $i=0$, displayed in the left part of Fig. 2. This approximation corresponds most closely to the spirit of the analytical result, Eq. (1b), in which only the scattering length appears, and indeed the correct asymptotic behavior can be observed for $k_{F} \rightarrow 0$, as it should. However, due to the fact that this interaction is unrealistically repulsive in momentum space, the gap decreases rapidly and finally disappears with increasing density. It is nevertheless worth mentioning that this type of contact interaction can be used without problems in the polarization part of the interaction, whereas it leads to divergencies when used naively as bare interaction in the BCS gap equation.

The result changes significantly when including the correct momentum dependence of the $T$ matrix to lowest order, according to Eq. (16): the gap (curve labeled $T$ ) remains now finite, but it is still strongly suppressed with respect to the BCS result. Only when further reducing the interaction by replacing the $T$ matrix scattering length with the result obtained from the $G$ matrix (curve labeled $G$ ), the ratio $\Delta_{2} / \Delta_{0}$ comes closer to the $k_{F} \rightarrow 0$ result, yet still remaining substantially below that limit at finite density.

Concerning the effect of the $p$ waves, we find that whether or not including the second (and third) term on the rhs of Eq. (13), the corresponding change of the result in Fig. 3 would be not discernible by the bare eye. Separate curves are therefore not shown. In the end, our final result is a 
reduction of the gap due to first-order polarization effects in the low-density region $k_{F}<0.05 \mathrm{fm}^{-1}$ by about a factor of 3 relative to the BCS value.

In the previous section we gave an estimate of the uncertainty of the present results with respect to the inclusion of higher-order polarization diagrams. The relative error $R$ was estimated in Eq. (21), and it is plotted in the middle panel of Fig. 3. It can be seen that this estimate becomes larger than $60 \%$ at the maximum Fermi momentum displayed, $k_{F}$ $=0.05 \mathrm{fm}^{-1}$, rapidly limiting the utility of the present approximation.

\section{CONCLUSIONS}

We studied the effects of correlations beyond the meanfield (BCS) approximation on the ${ }^{1} S_{0}$ pairing in low-density $\left(k_{F} \leq 0.05 \mathrm{fm}^{-1}\right)$ neutron matter. We performed an analysis of the in-medium interaction kernel to second order in the interaction and leading order in density (one hole line). The importance of considering the complete set of diagrams (direct and exchange) was demonstrated; in fact, the repulsive nature of the polarization interaction at low density results from the dominance of the exchange graphs compared to the direct polarization bubbles, see Eq. (11).

The analytically known low-density limit, Eq. (1b) (suppression of the BCS gap by a factor $\approx 2.2$ when $k_{F} \rightarrow 0$ ) was correctly reproduced, the numerical results indicating an even stronger suppression with increasing density. We found a surprisingly large effect due to the inclusion of Pauliblocking when replacing the $T$-matrix scattering length by the one obtained with the $G$ matrix. On the contrary, the influence of the $p$ waves turned out completely negligible in this density range.
The resulting net effect was a suppression of the gap relative to the BCS result by about a factor of 3 over the whole density region considered. One has therefore to conclude that the commonly used BCS approximation is not even reliable at very low density. The situation is different in the case of pairing with a positive scattering length, where at low density the BCS approximation describes the transition from pairing to the formation and Bose-Einstein condensation of bound states [19].

Unfortunately the present approach is limited to very low density, where the gap is actually extremely small [e.g., $\left.\Delta_{0}\left(k_{F}=0.01 \mathrm{fm}^{-1}\right) \approx 0.5 \times 10^{-6} \mathrm{MeV}\right]$, and cannot easily be extrapolated to more relevant higher density, where the modification of the gap could be very different, as is indeed predicted in some publications $[3,15]$. Even in the present work we were forced to make a number of approximations that are not well controlled. Apart from the approximate treatment of the integration appearing in Eq. (8), we completely neglected contributions to the interaction kernel of higher orders in the interaction and/or density, as well as any dispersive effects (energy dependence of interaction kernel and self-energy [26]) in the gap equation. All these are very difficult problems for the future, however.

\section{ACKNOWLEDGMENTS}

We acknowledge useful discussions with M. Baldo, J. Clark, G. Röpke, and P. Schuck. This work was supported in part by the programs "Estancias de científicos y tecnólogos extranjeros en España," SGR98-11 (Generalitat de Catalunya), and DGICYT (Spain) No. PB98-1247.
[1] S. L. Shapiro and S. A. Teukolsky, Black Holes, White Dwarfs, and Neutron Stars (Wiley, New York, 1983); M. A. Alpar, P. W. Anderson, D. Pines and J. Shaham, Astrophys. J. Lett. 249, L33 (1981); D. Pines, and M. A. Alpar, Nature (London) 316, 27 (1985); J. A. Sauls, in Timing Neutron Stars, edited by H. Ögelman and E. P. J. van den Heuvel (Kluwer, Dordrecht, 1989), p. 457; C. J. Pethick and D. G. Ravenhall, Annu. Rev. Astron. Astrophys. 45, 429 (1995); G. Lazzari and F. V. De Blasio, Nuovo Cimento A 108, 313 (1995); S. Tsuruta, Phys. Rep. 292, 1 (1998); H. Heiselberg and M. HjorthJensen, ibid. 328, 237 (2000).

[2] L. Amundsen and E. Østgaard, Nucl. Phys. A437, 487 (1985); M. Baldo, J. Cugnon, A. Lejeune, and U. Lombardo, ibid. A515, 409 (1990); A536, 349 (1991); T. Takatsuka and R. Tamagaki, Prog. Theor. Phys. Suppl. 112, 27 (1993); $\emptyset$. Elgarby and M. Hjorth-Jensen, Phys. Rev. C 57, 1174 (1998).

[3] J. M. C. Chen, J. W. Clark, R. D. Davé, and V. V. Khodel, Nucl. Phys. A555, 59 (1993).

[4] V. A. Khodel, V. V. Khodel, and J. W. Clark, Nucl. Phys. A598, 390 (1996).

[5] J. Bardeen, L. N. Cooper, and J. R. Schrieffer, Phys. Rev. 108, 1175 (1957).
[6] A. A. Abrikosov, L. P. Gorkov, and I. E. Dzyaloshinskii, Methods of Quantum Field Theory in Statistical Physics (Prentice-Hall, Englewood Cliffs, NJ, 1963).

[7] P. Nozières, Le Problème à N Corps (Dunod, Paris, 1963).

[8] J. R. Schrieffer, Theory of Superconductivity (AddisonWesley, New York, 1964).

[9] A. B. Migdal, Theory of Finite Systems and Applications to Atomic Nuclei (Benjamin, New York, 1964).

[10] A. L. Fetter and J. D. Walecka, Quantum Theory of ManyParticle Systems (McGraw-Hill, New York, 1971).

[11] D. Pines, in Proceedings of the 12th International Conference on Low Temperature Physics, edited by E. Kandu (Kligatu, Tokyo, 1971), p. 10.

[12] J. W. Clark, C.-G. Källman, C.-H. Yang, and D. A. Chakkalakal, Phys. Lett. 61B, 331 (1976).

[13] J. M. C. Chen, J. W. Clark, E. Krotschek, and R. A. Smith, Nucl. Phys. A451, 509 (1986).

[14] T. L. Ainsworth, J. Wambach, and D. Pines, Phys. Lett. B 222, 173 (1989); J. Wambach, T. L. Ainsworth, and D. Pines, Nucl. Phys. A555, 128 (1993).

[15] H.-J. Schulze, J. Cugnon, A. Lejeune, M. Baldo, and U. Lombardo, Phys. Lett. B 375, 1 (1996). 
[16] L. P. Gorkov and T. K. Melik-Barkhudarov, Sov. Phys. JETP 13, 1018 (1961).

[17] T. Papenbrock and G. F. Bertsch, Phys. Rev. C 59, 2052 (1999).

[18] H. Heiselberg, C. J. Pethick, H. Smith, and L. Viverit, Phys. Rev. Lett. 85, 2418 (2000).

[19] M. Baldo, U. Lombardo, and P. Schuck, Phys. Rev. C 52, 975 (1995); H. Stein, A. Schnell, T. Alm, and G. Röpke, Z. Phys. A 351, 295 (1995).

[20] S. A. Fayans and D. Zawischa, in Recent Progress in ManyBody Theories, edited by R. F. Bishop, K. A. Gernoth, N. R. Walet, and Y. Xian (World Scientific, Singapore, 2000), p. 403.

[21] R. B. Wiringa, V. G. J. Stoks, and R. Schiavilla, Phys. Rev. C 51, 38 (1995).

[22] W. Kohn and J. M. Luttinger, Phys. Rev. Lett. 15, 524
(1965).

[23] S. Babu and G. E. Brown, Ann. Phys. (N.Y.) 78, 1 (1973); O. Sjöberg, ibid. 78, 39 (1973); W. H. Dickhoff, A. Faessler, H. Müther, and Shi-Shu Wu, Nucl. Phys. A405, 534 (1983); S.-O. Bäckmann, G. E. Brown, and J. A. Niskanen, Phys. Rep. 124, 1 (1985); S.-O. Bäckmann, C.-G. Källman, and O. Sjöberg, Phys. Lett. 43B, 263 (1973); A. D. Jackson, E. Krotschek, D. E. Meltzer, and R. A. Smith, Nucl. Phys. A386, 125 (1982).

[24] H.-J. Schulze, A. Schnell, G. Röpke, and U. Lombardo, Phys. Rev. C 55, 3006 (1997).

[25] D. V. Efremov, M. S. Marenko, M. A. Baranov, and M. Y. Kagan, Sov. Phys. JETP 90, 861 (2000).

[26] P. Bozek, Nucl. Phys. A657, 187 (1999); Phys. Rev. C 62, 054316 (2000); M. Baldo and A. Grasso, Phys. Lett. B 485, 115 (2000). 